\title{
Mitochondrial haplotypes reveal olive fly (Bactrocera oleae) population substructure in the Mediterranean
}

Barbara van Asch • Isabel Pereira-Castro • Fernando Rei · Luís Teixeira da Costa

\begin{abstract}
The olive fly (Bactrocera oleae) is the most important olive tree (Olea europaea) pest. In the Mediterranean basin, where $98 \%$ of its main hosts are concentrated, it causes major agricultural losses, due to its negative effect on production and quality of both olive and olive oil. Previous phylogeographic analyses have established that Mediterranean olive fly populations are distinct from other Old World populations, but did not agree on the specific population substructure within this region. In order to achieve a higher resolution of the diversity of olive fly populations, particularly in Central and Western Mediterranean (home to $70 \%$ of the world production), we comparatively analyzed a set of samples from Portugal in the context of published mitochondrial sequences across the species' worldwide range. Strong evidence of population substructure was found in the Central and Western Mediterranean area, with two clearly separate phylogenetic branches. Together with previously published data, our results strongly support the existence of at least three distinct Mediterranean populations of the olive fly, raise the possibility of additional regional substructure and suggest specific avenues for future research. This knowledge can be instrumental in the development of better management and control strategies for a major pest of Mediterranean agriculture.
\end{abstract}

Keywords: Bactrocera oleae _ Mitochondrial_Mediterranean _ Phylogeography _ Population structure Olive fly 